\title{
Annular Bright Field Scanning Transmission Electron Microscopy Imaging Dynamics
}

S.D. Findlay, * N. Shibata, ${ }^{* * *}$ H. Sawada, ${ }^{* * *}$ E. Okunishi, $* * *$ Y. Kondo, ${ }^{* * *}$ S. Azuma, ${ }^{*}$ and Y. Ikuhara*,****,*****

* Institute of Engineering Innovation, the University of Tokyo, Tokyo 116-0013, Japan

** PRESTO, Japan Science and Technology Agency, Saitama, 332-0012, Japan

*** JEOL Ltd., Tokyo, 196-8558, Japan

**** Nanostructures Research Laboratory, Japan Fine Ceramics Center, Nagoya, 456-8587, Japan

***** WPI Advanced Institute for Materials Research, Tohoku University, Sendai, 980-8577, Japan

Annular bright field $(\mathrm{ABF})$ imaging, whereby an annular detector is positioned within the bright field region in an atomic resolution scanning transmission electron microscope, has recently been shown to produce images showing both light and heavy element columns simultaneously [1,2]. Using such bright field annular detectors has precedent [3], but atomic resolution ABF proves to have a reliability and robustness of interpretation for the simultaneous imaging of light and heavy columns that earlier work, prior to the development of aberration correctors and assuming the phase object approximation, did not appreciate. Fig. 1 shows a defocus-thickness map of ABF images of $\mathrm{TiO}_{2}$ [001]. The images (dark contrast at the column sites) provide directly interpretable structure information over a wide thickness range in a narrow band of defocus values centered about zero.

A simple s-state channeling model has been used to provide a basic explanation of the form and robustness of the $\mathrm{ABF}$ images [2]. However, that model is only approximate, providing some insight but not a detailed guide to the imaging dynamics. Systematic simulations are required for a fuller exploration of the imaging dynamics, allowing us to consider issues such as optimum aperture sizes and the effect of intercolumn spacing and structural distortion.

Figs. 2(a) and (b) plot the on-column signal as a function of thickness in a fictitious single-column structure assuming $22 \mathrm{mrad}$ and $32 \mathrm{mrad}$ probe-forming apertures respectively. There is some overlap of signal for different elements, suggesting that $\mathrm{ABF}$ contrast is not a completely reliable guide to column constituency, though greater discrimination between the elements is obtained for the larger probe-forming aperture. However, averaging the latter signal over thickness yields a monotonic relationship with atomic number. Fig. 2(c) plots this "signal", the zero thickness limit minus this average value, as a function of atomic number. The scaling is roughly $\mathrm{Z}^{1 / 3}$, though an exponential fit is better. Fig. 3 shows the effect of intercolumn spacing: closer columns leads to some displacement of the apparent column location, but the existence of the columns remains clear.

References

[1] E. Okunishi et al., Microsc. Microanal. 15(Suppl. 2) (2009) 164.

[2] S.D. Findlay et al., Appl. Phys. Lett. 95 (2009) 191913.

[3] J. Liu and J.M. Cowley, Ultramicroscopy 52 (1993) 335.

[4] S.D.F. was supported for part of this work as a Japan Society for the Promotion of Science (JSPS) fellow. N.S. acknowledges support from Industrial Technology Research Grant program in 2007 from New Energy and Industrial Technology Development Organization (NEDO) of Japan. 


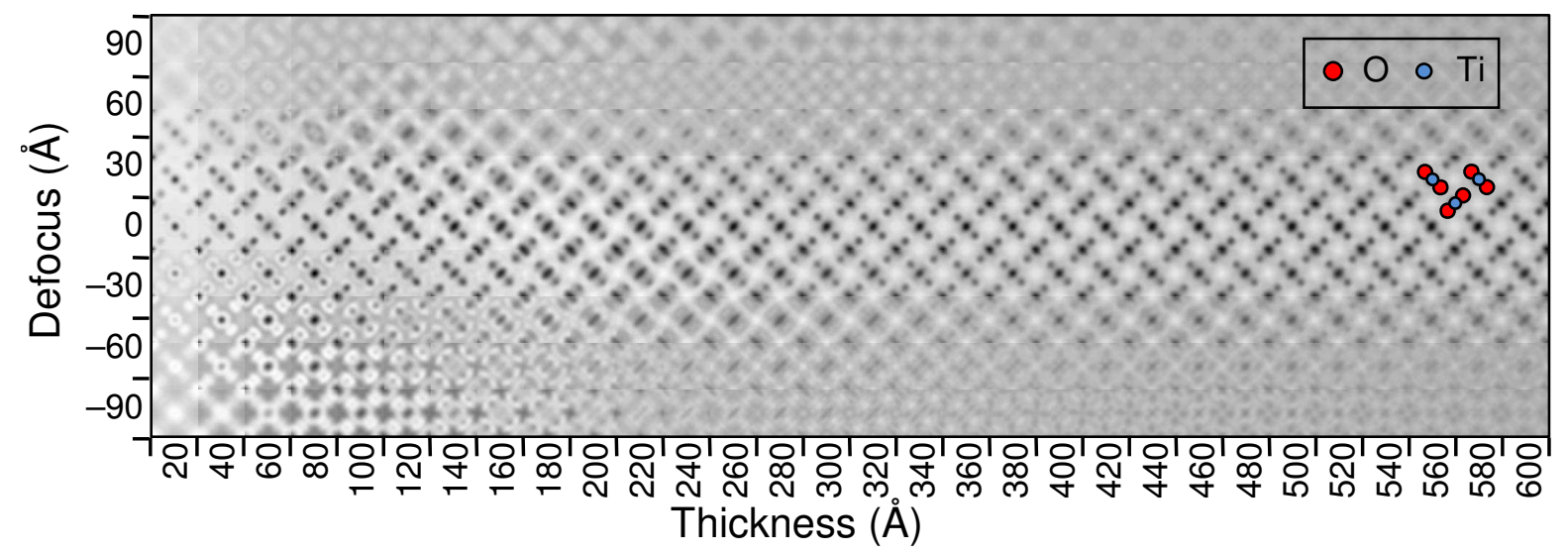

FIG. 1. Defocus-thickness map for the $\mathrm{ABF}$ image of $\mathrm{TiO}_{2}$ [001] assuming a $200 \mathrm{keV}$, aberrationfree, $22 \mathrm{mrad}$ aperture semiangle probe and a detector collection range of 11-22 mrad.

(a)

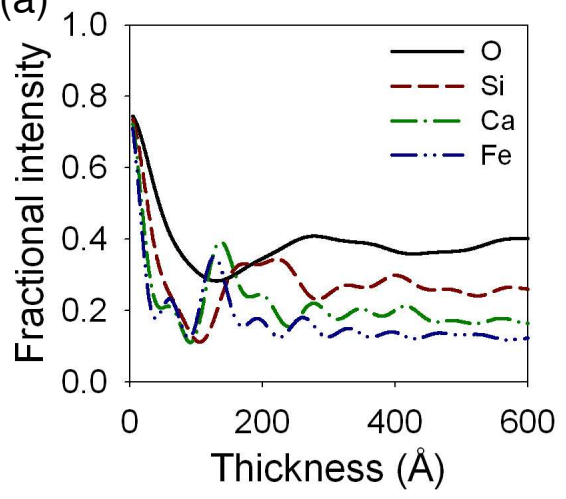

(b)

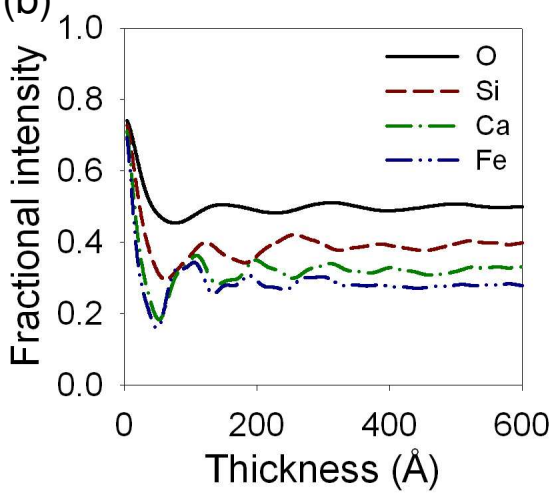

(c)

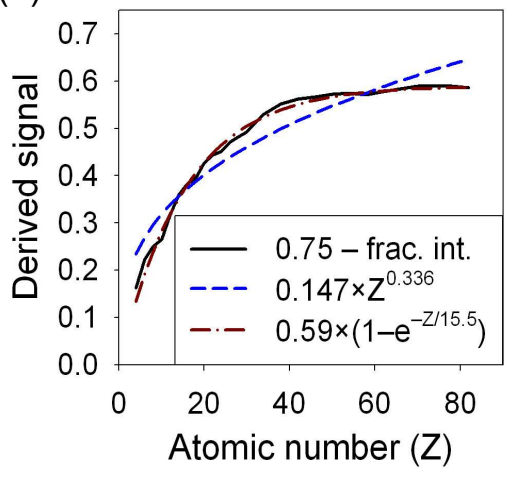

FIG. 2. ABF signal as a function of thickness with the probe on top of the column in a fictitious single-column structure for probe forming apertures $\alpha$ of (a) $22 \mathrm{mrad}$ and (b) $32 \mathrm{mrad}$, with ABF detector range $[\alpha / 2, \alpha]$ and for the elements labeled. (c) Zero-thickness limit less the averaged ABF signal over 300-600 $\AA$ as a function of atomic number, compared with approximate analytic forms.
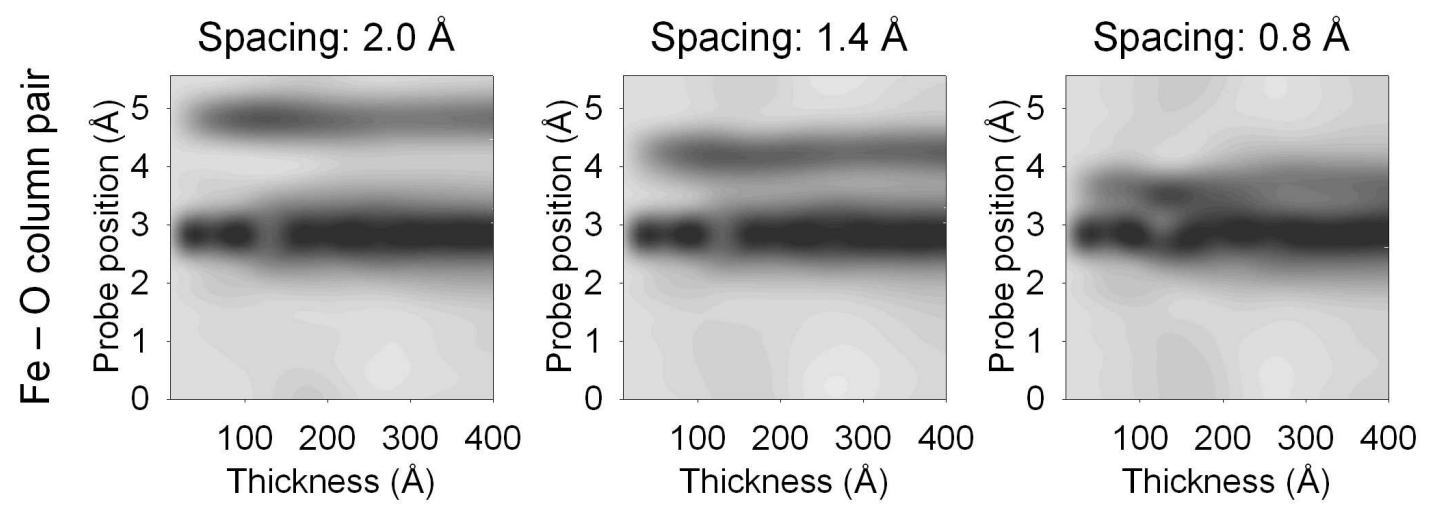

FIG. 3. ABF line scan as a function of specimen thickness for a fictitious two column (Fe and O) structure. The intercolumn spacing given above each panel. A $200 \mathrm{keV}$, aberration free, $22 \mathrm{mrad}$ aperture semiangle probe and a detector collection range of 11-22 mrad are assumed. 